\title{
A Complete Model of Crimean-Congo Hemorrhagic Fever (CCHF) Transmission Cycle with Nonlocal Fractional Derivative
}

\author{
Hakimeh Mohammadi $\mathbb{D}^{1}$ Mohammed K. A. Kaabar $\mathbb{D}^{2}{ }^{2}$ Jehad Alzabut $\mathbb{D},,^{3,4}$ \\ A. George Maria Selvam $\left(1,{ }^{5}\right.$ and Shahram Rezapour $\mathbb{1}^{6,7}$ \\ ${ }^{1}$ Department of Mathematics, Miandoab Branch, Islamic Azad University, Miandoab, Iran \\ ${ }^{2}$ Jabalia Camp, United Nations Relief and Works Agency (UNRWA) Palestinian Refugee Camp, Gaza Strip Jabalya, State of Palestine \\ ${ }^{3}$ Department of Mathematics and General Sciences, Prince Sultan University, Riyadh 11586, Saudi Arabia \\ ${ }^{4}$ Group of Mathematics, Faculty of Engineering, OSTIM Technical University, 06374 Ankara, Turkey \\ ${ }^{5}$ Department of Mathematics, Sacred Heart College (Autonomous), Tirupattur, Tamil Nadu 635601, India \\ ${ }^{6}$ Department of Mathematics, Azarbaijan Shahid Madani University, Tabriz, Iran \\ ${ }^{7}$ Department of Medical Research, China Medical University Hospital, China Medical University, Taichung, Taiwan
}

Correspondence should be addressed to Mohammed K. A. Kaabar; mohammed.kaabar@wsu.edu and Shahram Rezapour; rezapourshahram@yahoo.ca

Received 23 April 2021; Revised 23 May 2021; Accepted 14 June 2021; Published 29 June 2021

Academic Editor: Liliana Guran

Copyright (C) 2021 Hakimeh Mohammadi et al. This is an open access article distributed under the Creative Commons Attribution License, which permits unrestricted use, distribution, and reproduction in any medium, provided the original work is properly cited.

\begin{abstract}
Crimean-Congo hemorrhagic fever is a common disease between humans and animals that is transmitted to humans through infected ticks, contact with infected animals, and infected humans. In this paper, we present a boxed model for the transmission of Crimean-Congo fever virus. With the help of the fixed-point theory, our proposed system model is investigated in detail to prove its unique solution. Given that the Caputo fractional-order derivative preserves the system's historical memory, we use this fractional derivative in our modeling. The equilibrium points of the proposed system and their stability conditions are determined. Using the Euler method for the Caputo fractional-order derivative, we calculate the approximate solutions of the fractional system, and then, we present a numerical simulation for the transmission of Crimean-Congo hemorrhagic fever.
\end{abstract}

\section{Introduction}

Crimean-Congo hemorrhagic fever is a common disease between human and livestock. The virus that causes this disease is one of the most important Arthropod-Borne viruses of the Bunyaviridae family, and it is a genus of Nairovirus that can cause severe and deadly disease in humans, but it is not associated with any specific clinical sign in livestock. The most common vector is a tick called Hyalomma, but it is also transmitted by other ticks [1]. The average mortality rate among infected people is 30 percent [2].

The first known case of the disease was recorded in 1942 in the Crimean region of the former Soviet Union. The virus that caused the disease was also isolated from the blood of a feverish patient in 1956 in the Democratic Republic of the
Congo. The relationship between these two reported places of disease and the attention to the main symptoms of the disease (fever and bleeding) has led to the choice of the current name of the disease (Crimean-Congo hemorrhagic fever) $($ see $[3,4])$. The disease has been reported in more than 31 countries in Africa, Asia, and Eastern Europe [5].

Numerous serological studies have confirmed infections in animals, especially domestic animals such as cattle, sheep, and goats that may occur as feverish reactions. Infection in animals occurs through the bite of ticks infected with the Crimean-Congo hemorrhagic fever virus [6]. CrimeanCongo hemorrhagic fever virus can also infect a wide range of wild animals. Among wild mammals, rabbits have been an important reservoir of the virus in the European part of the former Soviet Union and Bulgaria. In Asia, hedgehogs, 
rats, and particular species of rabbits are the reservoir of this virus [7].

The most important ways of getting infected with the Crimean-Congo fever virus are as follows: the person is getting bitten by infected ticks, the contact of scratched or injured skin of a person's body with the contents of infected crushed ticks, the contact of damaged skin or human mucosa with infected animal blood or secretions, and the contact with blood and other secretions of the infected person, as well as the contact with infected surgical instruments [8-10]. Because Crimean-Congo hemorrhagic fever is more likely to come from the contact with an infected animal or human or bite by infected ticks are transmitted to humans so hunters, farmers, ranchers, health personnel, and those contact with infected animals and humans due to occupations more likely to be infected.

Clinical signs and the course of this disease include four stages:

(i) Incubation Period: After a tick bite, the incubation period usually lasts 1-3 days and reaches a maximum of 1 day. The incubation period following the contact with infected tissues or blood is usually 5-6 days, and the maximum time is 13 days [11]

(ii) Prehaemorrhagy: In 80 percent of cases, CrimeanCongo hemorrhagic fever infections are asymptomatic. People in whom the disease has clinical manifestations, the onset of symptoms is sudden, and it lasts about 1 up to 7 days (average 3 days). The initial symptoms are severe headache, fever, chills, joint pain, muscle cramps, dizziness, pain and stiffness of the neck, eye pain, and fear of light. Nausea, vomiting, diarrhea, abdominal pain, loss of appetite, swelling and redness of the face, decreased heart rate, and low blood pressure have also been reported [12]

(iii) Haemorrhagy: The bleeding phase is short and usually starts on days 3 to 5 and lasts 1 to 11 days (average 4 days). Bleeding in the mucosa, hematoma, bleeding gums and nose, bleeding from the uterus, bloody sputum, and bleeding from the conjunctiva and ears are the symptoms of the disease at this stage. Bleeding from various organs worsens the patient's condition so that the patient may die in the second week of severe bleeding, intravascular coagulation, liver failure, and dehydration [13]

(iv) Convalescence Period: Between days 7 and 20, the fever stops, and then the bleeding stops. From the tenth day, when the skin lesions fade, patients gradually recover. Most patients are discharged from the hospital in the third to sixth week after the onset of illness when blood and urine tests return to be normal [11]

Biological and mathematical researchers have conducted research studies to model the transmission of CrimeanCongo fever. Kashkynbayev et al. have used an SI Model to study tick-borne diseases, including Crimean-Congo fever
[14]. Ergena et al. have used an SIR Model to study the dynamic of tuberculosis and Crimean-Congo fever as epidemic diseases [15]. Switkes et al. have used the deterministic system of nonlinear differential equations to model the transmission of Crimean-Congo haemorrhagic fever with host immunity [16].

In recent years, extensive studies [17-19] have been conducted on the mathematical analysis of fractional derivatives and integrals. The fractional-order derivative is nonlocal and includes the historical and long-term memory effect of the system, and this is one of its most important advantages over the integer-order derivative, which helps to model natural phenomena better [20-23].

By the expansion of fractional differential calculus, researchers in many branches of science have turned to use the fractional differential equation system in their research. Mathematical modeling of the spread of viruses and the transmission of infectious diseases using systems of fractional differential equations are considered as one of the topics that has attracted the attention of researchers in recent decades [24]. Almeida et al. [25] proposed an epidemiologial MSEIR model formulated in the sense of Caputo fractional derivative. Baleanu et al. $[26,27]$ formulated new models of the HIV-1 infection of CD4+ T-cell and human liver via Caputo-Fabrizio fractional derivative. In addition, Rezapour et al. [28, 29] introduced new models for the spread of AH1N1 influenza and the transmission of Zika virus between humans and mosquitoes via Caputo-Fabrizio and Cuputo fractional derivatives, respectively. Singh analyzed the fractional blood alcohol model with a composite fractional derivative [30], and Singh et al. investigated the fractional fish farm model and fractional model of guava for biological pest control, [31, 32]. Also, Ghanbari et al. presented an efficient numerical method for the fractional model of allelopathic stimulatory phytoplankton species [33].

In this article, we model the complete Crimean-Congo fever transmission cycle between humans, animals, and ticks, which in previous articles, researchers have only modeled a part of the cycle. Due to the effect of fractional derivative memory and good results obtained in recent years from fractional mathematical modeling, in this study, we use the fractional-order differential equation system to model the Crimean-Congo fever transmission.

The structure of this paper is organized as follows: In Section 2, some basic definitions and concepts of fractional calculus are recalled. A fractional-order mathematical model for the Crimean-Congo fever transmission cycle is formulated in Section 3. In Section 4, with the help of the fixedpoint theory, our proposed system (10) is proven to have a unique solution. The approximate solution of the fractional differential equation system (10) is obtained numerically, and a numerical simulation for the transmission of the Crimean-Congo fever virus is also provided in Section 5. In Section 6, we conclude our research work.

\section{Preliminary Results and Definitions}

In the current section, we recall the two definitions of the fractional-order derivative and corresponding integral of 
each one. A concept of the Laplace transform of fractional derivative is also discussed.

Definition 1 [34]. For an integrable function $w$, the Caputo derivative of fractional order $\vartheta \in(0,1)$ is given by

$$
{ }^{C} D^{\vartheta} w(t)=\frac{1}{\Gamma(m-\vartheta)} \int_{0}^{t} \frac{w^{(m)}(v)}{(t-v)^{9-m+1}} d v, \quad m[\vartheta]+1
$$

The Gamma function, denoted by $\Gamma($.$) , is defined as:$

$$
\Gamma(\vartheta)=\lim _{m \longrightarrow \infty} \frac{m ! m^{\vartheta}}{\vartheta(\vartheta+1)(\vartheta+2) \cdots(\vartheta+m)} .
$$

Also, the corresponding fractional integral of order $\vartheta$ with $\operatorname{Re}(\vartheta)>0$ is given by

$$
{ }^{C} I^{\vartheta} w(t)=\frac{1}{\Gamma(\vartheta)} \int_{0}^{t}(t-v)^{9-1} w(v) d v
$$

Definition 2 ([35, 36]). For $w \in H^{1}(c, d)$ and $d>c$, the Caputo-Fabrizio derivative of fractional order $\vartheta \in(0,1)$ for $w$ is given by

$$
{ }^{C F} D^{\vartheta} w(t)=\frac{M(\vartheta)}{(1-\vartheta)} \int_{c}^{t} \exp \left(\frac{-\vartheta}{1-\vartheta}(t-v)\right) w^{\prime}(v) d v
$$

where $t \geq 0, M(\vartheta)$ is a normalization function that depends on $\vartheta$ and $M(0)=M(1)=1$. If $w \notin H^{1}(c, d)$ and $0<\vartheta<1$, this derivative for $w \in L^{1}(-\infty, d)$ as given by

${ }^{C F} D^{\vartheta} w(t)=\frac{\vartheta M(\vartheta)}{(1-\vartheta)} \int_{-\infty}^{d}(w(t)-w(v)) \exp \left(\frac{-\vartheta}{1-\vartheta}(t-v)\right) d v$

Also, the corresponding $C F$ fractional integral is presented by

$$
{ }^{C F} I^{\vartheta} w(t)=\frac{2(1-\vartheta)}{(2-\vartheta) M(\vartheta)} w(t)+\frac{2 \vartheta}{(2-\vartheta) M(\vartheta)} \int_{0}^{t} w(v) d v
$$

The Laplace transform is one of the most important tools in solving differential equations, which has different definitions in fractional calculus. The next definition presents the Laplace transform of the Caputo fractional-order derivative.

Definition 3 [34]. The Laplace transform of Caputo Fractional differential operator of order $\vartheta$ is given by

$$
L\left[{ }^{C} D^{\vartheta} w(t)\right](s)=s^{\vartheta} L w(t)-\sum_{i=0}^{m-1} s^{9-i-1} w^{(i)}(0), \quad m-1<\vartheta \leq m \in N .
$$

This can also be obtained in the following form:

$$
L\left[{ }^{C} D^{9} w(t)\right]=\frac{s^{m} L[w(t)]-s^{m-1} w(0)-s^{m-1} w^{\prime}(0)-\cdots-w^{(m-1)}}{s^{m-9}} .
$$

\section{Model Formulation}

Mathematical models are considered as one of the most important tools in the study of disease transmission. In this section, we present a fractional-order mathematical model for the Crimean-Congo fever transmission cycle.

Crimean-Congo haemorrhagic fever (CCHF) is a feverish hemorrhagic disease that is mostly transmitted by ticks. Although the virus is specific to animals, single infection, and epidemic cases of CCHF also occurred in humans. To model the transmission of this viral disease, we consider the population of transmitting ticks $N_{k}$, the population of livestock and wild animals $N_{l}$, and the human population $N_{h}$. We divide the tick population into two groups and denote susceptible ticks with $S_{k}$ and infected ticks with $I_{k}$. In the previous section, we have mentioned that livestock and some wild animals can also be infected with this disease and be a virus reservoir, which we divide into two groups, susceptible group $S_{l}$ and infected group $I_{l}$. Like the previous two populations, we divide the human population into two susceptible $S_{h}$ and infected $I_{h}$ groups. Susceptible ticks are infected through infected ticks at the effective contact rate $\beta_{1}$ and through infected animals at the effective contact rate $\beta_{2}$. Infected ticks transmit the virus to susceptible animals at the effective contact rate $\beta_{3}$ when they feed on animal body. Crimean-Congo fever virus is transmitted to humans in three ways. The virus is transmitted to humans through infected ticks at the effective contact rate $\beta_{4}$, through the blood and blood products of an infected animal at the effective contact rate $\beta_{5}$, and through the blood and bloody mucosa of infected human at the effective contact rate $\beta_{6}$. We also consider the recruitment rate of ticks, animals, and humans as $\Lambda_{k}, \Lambda_{l}$, and $\Lambda_{h}$, respectively. The natural mortality rates of ticks, animals, and humans are $d_{k}, d_{l}$, and $d_{h}$, respectively.

Based on the provided explanations, we present the Crimean-Congo fever transfer model with the system of differential equations as follows:

$$
\left(\begin{array}{l}
\frac{d S_{k}}{d t}=\Lambda_{k}-\beta_{1} S_{k}(t) I_{k}(t)-\beta_{2} S_{k}(t) I_{l}(t)-d_{k} S_{k}(t) \\
\frac{d I_{k}}{d t}=\beta_{1} S_{k}(t) I_{k}(t)+\beta_{2} S_{k}(t) I_{l}(t)-d_{k} I_{k}(t) \\
\frac{d S_{l}}{d t}=\Lambda_{l}-\beta_{3} S_{l}(t) I_{l}(t)-d_{l} S_{l}(t) \\
\frac{d I_{l}}{d t}=\beta_{3} S_{l}(t) I_{l}(t)-d_{l} I_{l}(t) \\
\frac{d S_{h}}{d t}=\Lambda_{h}-\beta_{4} S_{h}(t) I_{k}(t)-\beta_{5} S_{h}(t) I_{l}(t)-\beta_{6} S_{h}(t) I_{h}(t)-d_{h} S_{h}(t) \\
\frac{d I_{h}}{d t}=\beta_{4} S_{h}(t) I_{k}(t)+\beta_{5} S_{h}(t) I_{l}(t)+\beta_{6} S_{h}(t) I_{h}(t)-d_{h} I_{h}(t)
\end{array}\right.
$$


where all of the initial conditions $S_{k}(0)=S_{0 k}, I_{k}(0)=I_{0 k}, S_{l}($ $0)=S_{0 l}, I_{l}(0)=I_{0 l}, S_{h}(0)=S_{0 h}$, and $I_{h}(0)=I_{0 h}$ are positive.

The fractional-order system (FDEs) is related to systems with memory, history, or nonlocal effects which exist in the many biological systems that show the realistic biphasic decline behavior of infection or diseases but at a slower rate. In the above integer-order system, since the internal memory effects of the biological system of CCHF are not included, it is better that we extend the proposed ordinary model to a fractional model. In this alternative, the equality of the dimensions of both sides of the equation is disturbed, and we use an auxiliary parameter $\sigma$, with the dimension of sec., to solve this problem ([37]). Thus, the fractional-order model for the Crimean-Congo haemorrhagic fever (CCHF) is given as follows:

$$
\left\{\begin{array}{l}
\sigma^{9-1 C} D_{t}^{9} S_{k}(t)=\Lambda_{k}-\beta_{1} S_{k}(t) I_{k}(t)-\beta_{2} S_{k}(t) I_{l}(t)-d_{k} S_{k}(t), \\
\sigma^{9-1 C} D_{t}^{9} I_{k}(t)=\beta_{1} S_{k}(t) I_{k}(t)+\beta_{2} S_{k}(t) I_{l}(t)-d_{k} I_{k}(t), \\
\sigma^{9-1 C} D_{t}^{9} S_{l}(t)=\Lambda_{l}-\beta_{3} S_{l}(t) I_{k}(t)-d_{l} S_{l}(t), \\
\sigma^{9-1 C} D_{t}^{9} I_{l}(t)=\beta_{3} S_{l}(t) I_{k}(t)-d_{l} I_{l}(t), \\
\sigma^{9-1 C} D_{t}^{9} S_{h}(t)=\Lambda_{h}-\beta_{4} S_{h}(t) I_{k}(t)-\beta_{5} S_{h}(t) I_{l}(t)-\beta_{6} S_{h}(t) I_{h}(t)-d_{h} S_{h}(t), \\
\sigma^{9-1 C} D_{t}^{9} I_{h}(t)=\beta_{4} S_{h}(t) I_{k}(t)+\beta_{5} S_{h}(t) I_{l}(t)+\beta_{6} S_{h}(t) I_{h}(t)-d_{h} I_{h}(t),
\end{array}\right.
$$

where $t \geq 0$ and $0<\vartheta<1$.

3.1. Nonnegative Solution. To show the nonnegativity of solutions, we claim that $M=\left\{\left(S_{k}, I_{k}, S_{l}, I_{l}, S_{h}, I_{h}\right) \in R_{6}^{+}: N_{k}(t) \leq\right.$ $\left.\left(\Lambda_{k} / d_{k}\right), N_{l}(t) \leq\left(\Lambda_{l} / d_{l}\right), N_{h}(t) \leq\left(\Lambda_{h} / d_{h}\right)\right\}$ is the feasibility region of system (10). To prove this claim, we consider the following Lemma.

Lemma 4. The closed set $M$ with respect to the fractional system (10) is positively invariant.

Proof. We first add two relations in the system (10) to obtain the fractional derivative of the total population of ticks. So,

$$
\sigma^{9-1 C} D_{t}^{v} N_{k}(t)=\Lambda_{k}-d_{k} N_{k}(t)
$$

where $N_{k}(t)=S_{k}(t)+I_{k}(t)$. We apply the Laplace transform to the parties of the above relation, then

$$
N_{k}(t)=N_{k}(0) E_{\vartheta}\left(-d_{k} \sigma^{1-\vartheta} t^{\vartheta}\right)+\int_{0}^{t} \Lambda_{k} \sigma^{1-\vartheta} \eta^{9-1} E_{\vartheta, \vartheta}\left(-d_{k} \sigma^{1-\eta} \eta^{\vartheta}\right) d \eta .
$$

In the above equation, $N_{k}(0)$ is the initial size of ticks population, and the terms $E_{\vartheta}, E_{\vartheta, \vartheta}$ are the Mittag-Leffler functions which are defined by

$$
E_{\vartheta}(w)=\sum_{n=0}^{\infty} \frac{w^{n}}{\Gamma(1+n \vartheta)}, E_{\vartheta, \vartheta}(w)=\sum_{n=0}^{\infty} \frac{w^{n}}{\Gamma(\vartheta+n \vartheta)}, \quad \vartheta>0 .
$$

By simplifying the relations, we conclude that

$$
\begin{aligned}
N_{k}(t)= & N_{k}(0) E_{\vartheta}\left(-d_{k} \sigma^{1-\vartheta} t^{\vartheta}\right)+\int_{0}^{t} \Lambda_{k} \sigma^{1-\vartheta} \eta^{\vartheta-1} \\
& \cdot \sum_{n=0}^{\infty} \frac{(-1)^{n} d_{k}^{n} \sigma^{n(1-\vartheta)} \eta^{n \vartheta}}{\Gamma(n \vartheta+\vartheta)} d \eta=\frac{\Lambda_{k} \sigma^{1-\vartheta}}{d_{k} \sigma^{1-\vartheta}} \\
& +E_{\vartheta}\left(-d_{k} \sigma^{1-\vartheta} t^{\vartheta}\right)\left(N_{k}(0)-\frac{\Lambda_{k} \sigma^{1-\vartheta}}{d_{k} \sigma^{1-\vartheta}}\right), \\
= & \frac{\Lambda_{k}}{d_{k}}+E_{\vartheta}\left(-d_{k} \sigma^{1-\vartheta} t^{\vartheta}\right)\left(N_{k}(0)-\frac{\Lambda_{k}}{d_{k}}\right) .
\end{aligned}
$$

Now, if $N_{k}(0) \leq\left(\Lambda_{k} / d_{k}\right)$, then for $t>0, N_{k}(t) \leq\left(\Lambda_{k} / d_{k}\right)$. At the same way for $N_{l}$ and $N_{h}$, we can prove that if $N_{l}(0)$ $\leq\left(\Lambda_{l} / d_{l}\right)$ and $N_{h}(0) \leq\left(\Lambda_{h} / d_{h}\right)$, then $N_{l}(t) \leq\left(\Lambda_{l} / d_{l}\right)$ and $N_{h}(t) \leq\left(\Lambda_{h} / d_{h}\right)$. Thus, the closed set $M$ with respect to fractional model (2) is positively invariant.

3.2. Equilibrium Points. In the current section, we determine the equilibrium points of the system (10) and the basic reproduction number. We present the necessary conditions for the stability of the system at the equilibrium point. To determine the equilibrium points, we set the equations to zero in system (10),

$$
\begin{aligned}
{ }^{C} D^{\vartheta} S_{k}(t) & ={ }^{C} D^{\vartheta} I_{k}(t)={ }^{C} D^{\vartheta} S_{l}(t)={ }^{C} D^{\vartheta} I_{l}(t)={ }^{C} D^{\vartheta} S_{h}(t) \\
& ={ }^{C} D^{\vartheta} I_{h}(t)=0,
\end{aligned}
$$

We solve the resulting algebraic equations and determine the equilibrium point of the system. The disease-free equilibrium point, denoted by $E_{0}$, is obtained as: $E_{0}=\left(\left(\Lambda_{k}\right)\right.$ $\left.\left.d_{k}\right), 0,\left(\Lambda_{l} / d_{l}\right), 0,\left(\Lambda_{h} / d_{h}\right), 0\right)$. The second equilibrium point, called the endemic equilibrium point, is obtained as $E^{*}=$ $\left(S_{k}^{*}, I_{k}^{*}, S_{l}^{*}, I_{l}^{*}, S_{h}^{*}, I_{h}^{*}\right)$,

$$
\begin{aligned}
S_{k}^{*} & =\frac{\Lambda_{k}}{\beta_{1} I_{k}^{*}+\beta_{2} I_{l}^{*}+d_{k}}, S_{l}^{*}=\frac{\Lambda_{l}}{\beta_{3} I_{k}^{*}+d_{l}}, S_{h}^{*} \\
& =\frac{\Lambda_{h}}{\beta_{4} I_{k}^{*}+\beta_{5} I_{l}^{*}+\beta_{6} I_{h}^{*}+d_{h}} .
\end{aligned}
$$

When the basic reproduction number is greater than one, and the spread of the disease continues, the endemic equilibrium point is defined. To obtain the basic reproduction number, we use the next generation method [38]. We consider the matrix form of the system (10) as follows:

$$
{ }^{C} D^{9} v(t)=F(v(t))-V(v(t)),
$$


where

$$
\begin{gathered}
F(v(t))=\sigma^{1-9}\left[\begin{array}{c}
\beta_{1} S_{k}(t) I_{k}(t)+\beta_{2} S_{k}(t) I_{l}(t) \\
\beta_{3} S_{l}(t) I_{k}(t) \\
\beta_{4} S_{h}(t) I_{k}(t)+\beta_{5} S_{h}(t) I_{l}(t)+\beta_{6} S_{h}(t) I_{h}(t)
\end{array}\right], \\
V(v(t))=\sigma^{1-9}\left[\begin{array}{c}
d_{k} I_{k}(t) \\
d_{l} I_{l}(t) \\
d_{h} I_{h}(t)
\end{array}\right] .
\end{gathered}
$$

By calculating the Jacobian matrix for $F$ and $V$ at the disease-free equilibrium point, we obtain:

$$
\begin{aligned}
& J_{F}\left(E_{0}\right)=\sigma^{1-9}\left[\begin{array}{ccc}
\frac{\beta_{1} \Lambda_{k}}{d_{k}} & \frac{\beta_{2} \Lambda_{k}}{d_{k}} & 0 \\
\frac{\beta_{3} \Lambda_{l}}{d_{l}} & 0 & 0 \\
\frac{\beta_{4} \Lambda_{h}}{d_{h}} & \frac{\beta_{5} \Lambda_{h}}{d_{h}} & \frac{\beta_{6} \Lambda_{h}}{d_{h}}
\end{array}\right], \\
& J_{v}\left(E_{0}\right)=\sigma^{1-9}\left[\begin{array}{ccc}
d_{k} & 0 & 0 \\
0 & d_{l} & 0 \\
0 & 0 & d_{h}
\end{array}\right] .
\end{aligned}
$$

The basic reproduction number $R_{0}$ is defined as the eigenvalue of next generation matrix of system (10), $R_{0}=$ $\rho\left(F V^{-1}\right)$. We obtain: $R_{0}=\max \left(R_{h}, R_{k l}\right)$,

$$
R_{h}=\frac{\beta_{6} \Lambda_{h}}{d_{h}^{2}}, R_{k l}=\frac{\beta_{1} \Lambda_{k} d_{l}+\sqrt{\beta_{1}^{2} \Lambda_{k}^{2} d_{l}^{2}+4 \beta_{2} \beta_{3} \Lambda_{k} \Lambda_{l} d_{k}^{2}}}{2 d_{l} d_{k}^{2}} .
$$

In commonly used infection models, when $R_{0}>1$, the infection will be able to start spreading in a population, but not if $R_{0}<1$.

3.3. Stability of Equilibrium Points. To determine the necessary conditions for the stability of the disease-free equilibrium point, we investigate the roots of the characteristic equation of system (10). The Jacobian matrix of the system (10) is

$$
J=\sigma^{1-9} \times\left[\begin{array}{cccccc}
-\beta_{1} I_{k}-\beta_{2} I_{l}-d_{k} & -\beta_{1} S_{k} & 0 & -\beta_{2} S_{k} & 0 & 0 \\
\beta_{1} I_{k}+\beta_{2} I_{l} & \beta_{1} S_{k}-d_{k} & 0 & \beta_{2} S_{k} & 0 & 0 \\
0 & -\beta_{3} S_{l} & -\beta_{3} I_{k}-d_{l} & 0 & 0 & 0 \\
0 & \beta_{3} S_{l} & \beta_{3} I_{k} & -d_{l} & 0 & 0 \\
0 & -\beta_{4} S_{h} & 0 & -\beta_{5} S_{h} & -\beta_{4} I_{k}-\beta_{5} I_{l}-\beta_{6} I_{h}-d_{h} & -\beta_{h} S_{h} \\
0 & \beta_{4} S_{h} & 0 & \beta_{5} S_{h} & \beta_{4} I_{k}+\beta_{5} I_{l}+\beta_{6} I_{h} & \beta_{6} S_{h}-d_{h}
\end{array}\right]
$$

Then, the Jacobian matrix at $E_{0}$ is obtained as:

$$
J\left(E_{0}\right)=\sigma^{1-9}\left[\begin{array}{cccccc}
-d_{k} & -\beta_{1} \frac{\Lambda_{k}}{d_{k}} & 0 & -\beta_{2} \frac{\Lambda_{k}}{d_{k}} & 0 & 0 \\
0 & \beta_{1} \frac{\Lambda_{k}}{d_{k}}-d_{k} & 0 & \beta_{2} \frac{\Lambda_{k}}{d_{k}} & 0 & 0 \\
0 & -\beta_{3} \frac{\Lambda_{l}}{d_{l}} & -d_{l} & 0 & 0 & 0 \\
0 & \beta_{3} \frac{\Lambda_{l}}{d_{l}} & 0 & -d_{l} & 0 & 0 \\
0 & -\beta_{4} \frac{\Lambda_{h}}{d_{h}} & 0 & -\beta_{5} \frac{\Lambda_{h}}{d_{h}} & -d_{h} & -\beta_{h} \frac{\Lambda_{h}}{d_{h}} \\
0 & \beta_{4} \frac{\Lambda_{h}}{d_{h}} & 0 & \beta_{5} \frac{\Lambda_{h}}{d_{h}} & 0 & \beta_{6} \frac{\Lambda_{h}}{d_{h}}-d_{h}
\end{array}\right] .
$$

In the following theorem, we determine the necessary conditions for the stability of the disease-free equilibrium point.

Theorem 5. The disease-free equilibrium point $E^{0}$ is locally asymptotically stable if $R_{0}<1$.

Proof. The characteristic equation of matrix $J\left(E_{0}\right)$ is obtained as follows:

$$
\begin{aligned}
& \left(d_{l}+\lambda\right)\left(d_{k}+\lambda\right)\left(d_{h}+\lambda\right)\left(\beta_{6} \frac{\Lambda_{h}}{d_{h}}-d_{h}-\lambda\right) \\
& \cdot\left[\left(d_{l}+\lambda\right)\left(\beta_{1} \frac{\Lambda_{k}}{d_{k}}-d_{k}-\lambda\right)-\beta_{2} \beta_{3} \frac{\Lambda_{k} \Lambda_{l}}{d_{k} d_{l}}\right]=0 .
\end{aligned}
$$

Therefore, the eigenvalues of the Jacobin matrix are $\lambda_{1}=$ $-d_{l}, \lambda_{2}=-d_{k}, \lambda_{3}=-d_{h}, \lambda_{4}=\left(\beta_{6} \Lambda_{h} / d_{h}\right)-d_{h}$, and the roots of the following equation are:

$$
\lambda^{2}-\lambda\left(\frac{\beta_{1} \Lambda_{k}}{d_{k}}-d_{k}-d_{l}\right)-\frac{\beta_{1} \Lambda_{k} d_{l}}{d_{k}}+d_{l} d_{k}+\beta_{2} \beta_{3} \frac{\Lambda_{k} \Lambda_{l}}{d_{k} d_{l}}=0 .
$$

The three roots $\lambda_{1}, \lambda_{2}$, and $\lambda_{3}$ are negative. If $R_{0}<1$, then $R_{h}=\left(\beta_{6} \Lambda_{h} / d_{h}^{2}\right)<1$, we obtain $\lambda_{4}<0$. It also follows from $R_{0}$ $<1$ that $R_{k l}<1$, then we conclude by simplifying $\beta_{1} \Lambda_{k}<d_{k}^{2}$. In Equation (24), which is a quadratic equation, we have:

$$
P=\frac{-\beta_{1} \Lambda_{k} d_{l}}{d_{k}}+d_{l} d_{k}+\beta_{2} \beta_{3} \frac{\Lambda_{k} \Lambda_{l}}{d_{k} d_{l}}, S=\frac{\beta_{1} \Lambda_{k}}{d_{k}}-d_{k}-d_{l},
$$

since $\beta_{1} \Lambda_{k}<d_{k}^{2}$ then $P>0, S<0$ so Equation (24) has 2 negative roots. Therefore, all of the eigenvalues are negative, and the disease-free equilibrium point is locally asymptotically stable. 


\section{Existence of Unique Solution}

In the current section, using the fixed-point theory, we prove that system (10) has a unique solution. Fixed-point theory is essential in proving the existence of a solution to the proposed system where adequate conditions are provided by fixed-point theorems such that a unique fixed point exists for a given function. To achieve this goal, we prove that kernels are satisfied under the Lipschitz condition, and they are contraction. Then, the existence of solution to the proposed system is constructed via fixed-point theorem. From the Lipschitz condition, the uniqueness of our obtained solution is proven when the obtained condition is satisfied. form:

First, we consider system (10) in the following compact

$$
\left\{\begin{array}{l}
\sigma^{9-1 C} D_{t}^{9} S_{k}(t)=R_{1}\left(t, S_{k}(t)\right), \\
\sigma^{9-1 C} D_{t}^{9} I_{k}(t)=R_{2}\left(t, I_{k}(t)\right), \\
\sigma^{9-1 C} D_{t}^{9} S_{l}(t)=R_{3}\left(t, S_{l}(t)\right), \\
\sigma^{9-1 C} D_{t}^{9} I_{l}(t)=R_{4}\left(t, I_{l}(t)\right), \\
\sigma^{9-1 C} D_{t}^{9} S_{h}(t)=R_{5}\left(t, S_{h}(t)\right), \\
\sigma^{9-1 C} D_{t}^{9} I_{h}(t)=R_{6}\left(t, I_{h}(t)\right) .
\end{array}\right.
$$

We apply the fractional-order integral to the parties of the above equations, so

$$
\left\{\begin{array}{l}
S_{k}(t)-S_{k}(0)=\frac{\sigma^{1-\vartheta}}{\Gamma(\vartheta)} \int_{0}^{t} R_{1}\left(\mu, S_{k}\right)(t-\mu)^{\vartheta-1} d \mu, \\
I_{k}(t)-I_{k}(0)=\frac{\sigma^{1-\vartheta}}{\Gamma(\vartheta)} \int_{0}^{t} R_{2}\left(\mu, I_{k}\right)(t-\mu)^{\vartheta-1} d \mu, \\
S_{l}(t)-S_{l}(0)=\frac{\sigma^{1-\vartheta}}{\Gamma(\vartheta)} \int_{0}^{t} R_{3}\left(\mu, S_{l}\right)(t-\mu)^{\vartheta-1} d \mu, \\
I_{l}(t)-I_{l}(0)=\frac{\sigma^{1-\vartheta}}{\Gamma(\vartheta)} \int_{0}^{t} R_{4}\left(\mu, I_{l}\right)(t-\mu)^{\vartheta-1} d \mu, \\
S_{h}(t)-S_{h}(0)=\frac{\sigma^{1-\vartheta}}{\Gamma(\vartheta)} \int_{0}^{t} R_{5}\left(\mu, S_{h}\right)(t-\mu)^{\vartheta-1} d \mu, \\
I_{h}(t)-I_{h}(0)=\frac{\sigma^{1-\vartheta}}{\Gamma(\vartheta)} \int_{0}^{t} R_{6}\left(\mu, I_{h}\right)(t-\mu)^{\vartheta-1} d \mu .
\end{array}\right.
$$

In the following, we prove that the kernels $R_{j}, j=1,2,3$, 4, 5, 6 are satisfied in the Lipschitz condition, and they are contraction.

Theorem 6. Kernel $R_{1}$ is satisfied in Lipschitz condition and contraction if we have:

$$
0 \leq \beta_{1} z_{1}+\beta_{2} z_{2}+d_{k}<1
$$

Proof. We can write for $S_{k}$ and $S_{1 k}$,

$$
\begin{aligned}
& \left\|R_{1}\left(t, S_{k}\right)-R_{1}\left(t, S_{1 k}\right)\right\| \\
& \quad=\left\|-\beta_{1} I_{k}\left(S_{k}-S_{1 k}\right)-\beta_{2} I_{l}\left(S_{k}-S_{1 k}\right)-d_{k}\left(S_{k}-S_{1 k}\right)\right\|, \\
& \quad \leq \beta_{1}\left\|I_{k}\right\|\left\|S_{k}-S_{k}\right\|+\beta_{2}\left\|I_{l}\right\|\left\|S_{k}-S_{1 k}\right\|+d_{k}\left\|S_{k}-S_{1 k}\right\|, \\
& \quad \leq\left(\beta_{1}\left\|I_{k}\right\|+\beta_{2}\left\|I_{l}\right\|+d_{k}\right)\left\|S_{k}-S_{1 k}\right\|, \\
& \quad \leq\left(\beta_{1} z_{1}+\beta_{2} z_{2}+d_{k}\right)\left\|S_{k}-S_{1 k}\right\| .
\end{aligned}
$$

Consider $e_{1}=\beta_{1} z_{1}+\beta_{2} z_{2}+d_{k}$, where $\left\|I_{k}(t)\right\| \leq z_{1}$ and $\|$ $I_{l} \| \leq z_{2}$, are bounded functions. We get:

$$
\left\|R_{1}\left(t, S_{k}\right)-R_{1}\left(t, S_{1 k}\right)\right\| \leq e_{1}\left\|S_{k}(t)-S_{1 k}(t)\right\|,
$$

if $0 \leq \beta_{1} z_{1}+\beta_{2} z_{2}+d_{k}<1$, then the kernel $R_{1}$ is satisfied in Lipschitz condition, and it is contraction.

In a similar way, we can show that the kernels $R_{j}, j=2$, $3,4,5,6$ are satisfied in the Lipschitz condition as follows:

$$
\left\{\begin{array}{l}
\left\|R_{2}\left(t, I_{k}\right)-R_{2}\left(t, I_{1 k}\right)\right\| \leq e_{2}\left\|I_{k}(t)-I_{1 k}(t)\right\|, \\
\left\|R_{3}\left(t, S_{l}\right)-R_{3}\left(t, S_{1 l}\right)\right\| \leq e_{3}\left\|S_{l}(t)-S_{1 l}(t)\right\|, \\
\left\|R_{4}\left(t, I_{l}\right)-R_{4}\left(t, I_{1 l}\right)\right\| \leq e_{4}\left\|I_{l}(t)-I_{1 l}(t)\right\|, \\
\left\|R_{5}\left(t, S_{h}\right)-R_{3}\left(t, S_{1 h}\right)\right\| \leq e_{5}\left\|S_{h}(t)-S_{1 h}(t)\right\|, \\
\left\|R_{6}\left(t, I_{h}\right)-R_{4}\left(t, I_{1 h}\right)\right\| \leq e_{6}\left\|I_{h}(t)-I_{1 h}(t)\right\|,
\end{array}\right.
$$

so that $e_{2}=\beta_{1} z_{4}+d_{k}, e_{3}=\beta_{3} z_{1}+d_{l}, e_{4}=d_{l}, e_{5}=\beta_{4} z_{1}+\beta_{5} z_{2}$ $+\beta_{6} z_{3}+d_{h}, e_{6}=\beta_{6} z_{6}+d_{h}$ are bounded functions where $\|$ $I_{h}(t)\left\|\leq z_{3},\right\| S_{k}(t)\left\|\leq z_{4},\right\| S_{l}(t) \| \leq z_{5}$, and $\left\|S_{h}(t)\right\| \leq z_{6}$. Also, if $0 \leq e_{j}<1, j=2,3,4,5,6$, then $R_{j}$ are contraction for $j=2$, $3,4,5,6$.

Based on system (27), we define:

$$
\begin{aligned}
A_{1 n}(t)= & S_{n k}(t)-S_{(n-1) k}(t)=\frac{\sigma^{1-\vartheta}}{\Gamma(\vartheta)} \int_{0}^{t}\left(R_{1}\left(\mu, S_{(n-1) k}\right)\right. \\
& \left.-R_{1}\left(\mu, S_{(n-2) k}\right)\right)(t-\mu)^{\vartheta-1} d \mu, \\
A_{2 n}(t)= & I_{n k}(t)-I_{(n-1) k}(t)=\frac{\sigma^{1-\vartheta}}{\Gamma(\vartheta)} \int_{0}^{t}\left(R_{2}\left(\mu, I_{(n-1) k}\right)\right. \\
& \left.-R_{2}\left(\mu, I_{(n-2) k}\right)\right)(t-\mu)^{\vartheta-1} d \mu, \\
A_{3 n}(t)= & S_{n l}(t)-S_{(n-1) l}(t)=\frac{\sigma^{1-\vartheta}}{\Gamma(\vartheta)} \int_{0}^{t}\left(R_{3}\left(\mu, S_{(n-1) l}\right)\right. \\
& \left.-R_{3}\left(\mu, S_{(n-2) l}\right)\right)(t-\mu)^{\vartheta-1} d \mu, \\
A_{4 n}(t)= & I_{n l}(t)-I_{(n-1) l}(t)=\frac{\sigma^{1-\vartheta}}{\Gamma(\vartheta)} \int_{0}^{t}\left(R_{4}\left(\mu, I_{(n-1) l}\right)\right. \\
& \left.\quad-R_{4}\left(\mu, I_{(n-2) l}\right)\right)(t-\mu)^{9-1} d \mu,
\end{aligned}
$$




$$
\begin{aligned}
A_{5 n}(t)= & S_{n h}(t)-S_{(n-1) h}(t)=\frac{\sigma^{1-9}}{\Gamma(\vartheta)} \int_{0}^{t}\left(R_{5}\left(\mu, S_{(n-1) h}\right)\right. \\
& \left.-R_{5}\left(\mu, S_{(n-2) h}\right)\right)(t-\mu)^{9-1} d \mu, \\
A_{6 n}(t)= & I_{n h}(t)-I_{(n-1) h}(t)=\frac{\sigma^{1-9}}{\Gamma(\vartheta)} \int_{0}^{t}\left(R_{6}\left(\mu, I_{(n-1) h}\right)\right. \\
& \left.-R_{6}\left(\mu, I_{(n-2) h}\right)\right)(t-\mu)^{9-1} d \mu,
\end{aligned}
$$

where $S_{k}(0)=S_{0 k}, I_{k}(0)=I_{0 k}, S_{l}(0)=S_{0 l}, I_{l}(0)=I_{0 l}, S_{h}(0)=$ $S_{0 h}$, and $I_{h}(0)=I_{0 h}$ are initial conditions. The norm of $A_{1 n}$ in the above system is expressed as follows:

$$
\begin{aligned}
\left\|A_{1 n}(t)\right\|= & \left\|S_{n k}(t)-S_{(n-1) k}(t)\right\| \leq \frac{\sigma^{1-9}}{\Gamma(\vartheta)} \int_{0}^{t} \| R_{1}\left(\mu, S_{(n-1) k}\right) \\
& \left.-R_{1}\left(\mu, S_{(n-2) k}\right)\right)(t-\mu)^{9-1} \| d \mu .
\end{aligned}
$$

According to the Lipschitz condition (30), we conclude

$$
\left\|A_{1 n}(t)\right\| \leq \frac{\sigma^{1-9}}{\Gamma(\vartheta)} e_{1} \int_{0}^{t}\left\|A_{1(n-1)}(\mu)\right\| d \mu .
$$

Similarly, we can prove that

$$
\begin{aligned}
\left\|A_{2 n}(t)\right\| & \leq \frac{\sigma^{1-9}}{\Gamma(\vartheta)} e_{2} \int_{0}^{t}\left\|A_{2(n-1)}(\mu)\right\| d \mu, \\
\left\|A_{3 n}(t)\right\| & \leq \frac{\sigma^{1-\vartheta}}{\Gamma(\vartheta)} e_{3} \int_{0}^{t}\left\|A_{3(n-1)}(\mu)\right\| d \mu, \\
\left\|A_{4 n}(t)\right\| & \leq \frac{\sigma^{1-9}}{\Gamma(\vartheta)} e_{4} \int_{0}^{t}\left\|A_{4(n-1)}(\mu)\right\| d \mu, \\
\left\|A_{5 n}(t)\right\| & \leq \frac{\sigma^{1-9}}{\Gamma(\vartheta)} e_{5} \int_{0}^{t}\left\|A_{5(n-1)}(\mu)\right\| d \mu, \\
\left\|A_{6 n}(t)\right\| & \leq \frac{\sigma^{1-9}}{\Gamma(\vartheta)} e_{6} \int_{0}^{t}\left\|A_{6(n-1)}(\mu)\right\| d \mu .
\end{aligned}
$$

Therefore, we get

$$
\begin{aligned}
& S_{n k}(t)=\sum_{i=1}^{n} A_{1 i}(t), I_{n k}(t)=\sum_{i=1}^{n} A_{2 i}(t), S_{n l}(t)=\sum_{i=1}^{n} A_{3 i}(t), \\
& I_{n l}(t)=\sum_{i=1}^{n} A_{4 i}(t), S_{n h}(t)=\sum_{i=1}^{n} A_{5 i}(t), I_{n h}(t)=\sum_{i=1}^{n} A_{6 i}(t) .
\end{aligned}
$$

In the next theorem, we prove the existence of solution by the fixed-point theorem.

Theorem 7. The Crimean-Congo fever transmission fractional-order model (10) has a solution, if there exists $t_{\varepsilon}$ such that

$$
\frac{\sigma^{1-9}}{\Gamma(\vartheta)} t_{\varepsilon} e_{i}<1
$$

Proof. By Equation (34) and Equation (46), we obtain

$$
\begin{aligned}
& \left\|A_{1 n}(t)\right\| \leq\left\|S_{n k}(0)\right\|\left[\frac{\sigma^{1-9}}{\Gamma(\vartheta)} e_{1} t\right]^{n},\left\|A_{2 n}(t)\right\| \leq\left\|I_{n k}(0)\right\|\left[\frac{\sigma^{1-9}}{\Gamma(\mathcal{9})} e_{2} t\right]^{n}, \\
& \left\|A_{3 n}(t)\right\| \leq\left\|S_{n l}(0)\right\|\left[\frac{\sigma^{1-9}}{\Gamma(\mathcal{9})} e_{3} t\right]^{n},\left\|A_{4 n}(t)\right\| \leq\left\|I_{n l}(0)\right\|\left[\frac{\sigma^{1-9}}{\Gamma(\mathcal{9})} e_{4} t\right]^{n}, \\
& \left\|A_{5 n}(t)\right\| \leq\left\|S_{n h}(0)\right\|\left[\frac{\sigma^{1-9}}{\Gamma(9)} e_{5} t\right]^{n},\left\|A_{6 n}(t)\right\| \leq\left\|I_{n h}(0)\right\|\left[\frac{\sigma^{1-9}}{\Gamma(\vartheta)} e_{6} t\right]^{n} .
\end{aligned}
$$

The above relations show that the system has a continuous solution. Now, it is sufficient to show that the above functions construct the solution for the fractional-order model (10). We consider the following relations:

$$
\begin{gathered}
S_{k}(t)-S_{k}(0)=S_{n k}(t)-\mathrm{U}_{1 n}(t), I_{k}(t)-I_{k}(0)=I_{n k}(t)-\mathrm{U}_{2 n}(t), \\
S_{l}(t)-S_{l}(0)=S_{n l}(t)-\mathrm{U}_{3 n}(t), I_{l}(t)-I_{l}(0)=I_{n l}(t)-\mathrm{U}_{4 n}(t), \\
S_{h}(t)-S_{h}(0)=S_{n h}(t)-\mathrm{U}_{5 n}(t), I_{h}(t)-I_{h}(0)=I_{n h}(t)-\mathrm{U}_{6 n}(t) .
\end{gathered}
$$

The norm of $\mathrm{U}_{1 n}(t)$ is obtained as follows:

$$
\begin{aligned}
\left\|\mathrm{U}_{1 n}(t)\right\| & =\left\|\frac{\sigma^{1-9}}{\Gamma(\mathcal{\vartheta})} \int_{0}^{t}\left(R_{1}\left(\mu, S_{k}\right)-R_{1}\left(\mu, S_{(n-1) k}\right)\right) d \mu\right\| \\
& \leq \frac{\sigma^{1-9}}{\Gamma(\vartheta)} \int_{0}^{t}\left\|R_{1}\left(\mu, S_{k}\right)-R_{1}\left(\mu, S_{(n-1) k}\right)\right\| d \mu \\
& \leq \frac{\sigma^{1-9}}{\Gamma(\mathcal{Y})} e_{1}\left\|S_{k}-S_{(n-1) k}\right\| t .
\end{aligned}
$$

By continuing this repetitive method, we conclude:

$$
\left\|\mathrm{U}_{1 n}(t)\right\| \leq\left[\frac{\sigma^{1-9}}{\Gamma(\vartheta)} t\right]^{n+1} e_{1}^{n+1} k .
$$

At $t_{\varepsilon}$, we have

$$
\left\|\mathrm{U}_{1 n}(t)\right\| \leq\left[\frac{\sigma^{1-\vartheta}}{\Gamma(\vartheta)} t_{\varepsilon}\right]^{n+1} e_{1}^{n+1} k .
$$

If we take limit on the recent relation as $n$ approaches to $\infty$, it results $\left\|\mathrm{U}_{1 n}(t)\right\| \longrightarrow 0$. Similarly, we conclude that $\|$ $\mathrm{B}_{j n}(t) \| \longrightarrow 0, j=2,3,4,5,6$, and the proof is complete.

To show the uniqueness of the solution of CCHF model, we consider that the fractional-order system (10) has another solution such as $\left(S_{1 k}(t), I_{1 k}(t), S_{1 l}(t), I_{1 l}(t), S_{1 h}(t), I_{1 h}(t)\right.$, then for $S_{k}, S_{1 k}$ can be written as: 


$$
S_{k}(t)-S_{1 k}(t)=\frac{\sigma^{1-\vartheta}}{\Gamma(\vartheta)} \int_{0}^{t}\left(R_{1}\left(\mu, S_{k}\right)-R_{1}\left(\mu, S_{1 k}\right)\right) d \mu .
$$

We take the norm on the above equation, so

$$
\left\|S_{k}(t)-S_{1 k}(t)\right\|=\frac{\sigma^{1-\vartheta}}{\Gamma(\vartheta)} \int_{0}^{t}\left\|R_{1}\left(\mu, S_{k}\right)-R_{1}\left(\mu, S_{1 k}\right)\right\| d \mu .
$$

By Lipschitz condition (30), we obtain:

$$
\left\|S_{k}(t)-S_{1 k}(t)\right\| \leq \frac{\sigma^{1-\vartheta}}{\Gamma(\vartheta)} e_{1} t\left\|S_{k}(t)-S_{1 k}(t)\right\| .
$$

Thus,

$$
\left\|S_{k}(t)-S_{1 k}(t)\right\|\left(1-\frac{\sigma^{1-\vartheta}}{\Gamma(\vartheta)} e_{1} t\right) \leq 0
$$

Theorem 8. The solution of fractional-order system (10) is unique when the following condition is met:

$$
1-\frac{\sigma^{1-\vartheta}}{\Gamma(\vartheta)} e_{1} t>0
$$

Proof. Assume that the condition (46) holds, in which case we conclude from (46) and (47) that $\left\|S_{k}(t)-S_{1 k}(t)\right\|=0$, and this shows that $S_{h}(t)=S_{1 h}(t)$. In the same way, similar relationships can be reached for $I_{k}, S_{l}, I_{l}, S_{h}, I_{h}$. This completes the proof.

\section{Numerical Simulation and Discussion}

In this section, we first obtain the approximate solution of the fractional differential equation system (10) by a numerical method, and then, we present a numerical simulation for the transmission of the Crimean-Congo fever virus.

5.1. Numerical Method. We use the fractional Euler method for Caputo derivative [39] to obtain the approximate solutions of the Crimean-Congo hemorrhagic fever virus transmission model. First, we consider the compact form of the system (10) as follows:

$$
\sigma^{9-1 C} D_{t}^{\vartheta} \varphi(t)=Q(t, \varphi(t)), \varphi(0)=\varphi_{0}, 0 \leq t \leq T<\infty,
$$

where $\varphi=\left(S_{k}, I_{k}, S_{l}, I_{l}, S_{h}, I_{h}\right) \in R_{+}^{6}, \varphi_{0}=\left(S_{0 k}, I_{0 k}, S_{0 l}, I_{0 l}, S_{0 h}\right.$, $\left.I_{0 h}\right)$, and $Q(t)$ is a continuous real vector function that is satisfied in the Lipschitz condition as follows:

$$
\left\|Q\left(\varphi_{1}(t)\right)-Q\left(\varphi_{2}(t)\right)\right\| \leq m\left\|\varphi_{1}(t)-\varphi_{2}(t)\right\|, m>0 .
$$

We apply the fractional-order integral operator corresponding to the Caputo fractional-order derivative on both sides of Equation (48), so

$$
\varphi(t)=\sigma^{1-\vartheta}\left[\varphi_{0}+I^{\vartheta} Q(\varphi(t))\right], 0 \leq t \leq T<\infty .
$$

Set $r=(T-0) / N$ and $t_{n}=n r$, where $t \in[0, T]$ and $N$ is a natural number and $n=0,1,2, \cdots, N$. Let $\varphi_{n}$ be the approximation of $\varphi(t)$ at $t=t_{n}$. By the fractional Euler method ([39]), we get:

$$
\begin{aligned}
\varphi_{n+1} & =\sigma^{1-\vartheta}\left[\varphi_{0}+\frac{r^{\vartheta}}{\Gamma(\vartheta+1)} \sum_{p=0}^{n} \omega_{n+1, p} Q\left(t_{p}, \varphi_{p}\right)\right], p \\
& =0,1,2, \cdots, N-1,
\end{aligned}
$$

where

$$
\omega_{n+1, p}=(n+1-p)^{9}-(n-p)^{9}, p=0,1,2, \cdots, n .
$$

The obtained scheme is stable. Details of the stability analysis are given in Theorem (3.1) of [39]. According to the explanations provided, the answer of the system is obtained as follows:

$$
\begin{aligned}
& S_{(n+1) k}=\sigma^{1-\vartheta}\left[S_{0 k}+\frac{r^{\vartheta}}{\Gamma(\vartheta+1)} \sum_{p=0}^{n} \omega_{n+1, p} y_{1}\left(t_{p}, \varphi_{p}\right)\right], \\
& I_{(n+1) k}=\sigma^{1-\vartheta}\left[I_{0 k}+\frac{r^{\vartheta}}{\Gamma(\vartheta+1)} \sum_{p=0}^{n} \omega_{n+1, p} y_{2}\left(t_{p}, \varphi_{p}\right)\right], \\
& S_{(n+1) l}=\sigma^{1-\vartheta}\left[S_{0 l}+\frac{r^{\vartheta}}{\Gamma(\vartheta+1)} \sum_{p=0}^{n} \omega_{n+1, p} y_{3}\left(t_{p}, \varphi_{p}\right)\right], \\
& I_{(n+1) l}=\sigma^{1-\vartheta}\left[I_{0 l}+\frac{r^{\vartheta}}{\Gamma(\vartheta+1)} \sum_{p=0}^{n} \omega_{n+1, p} y_{4}\left(t_{p}, \varphi_{p}\right)\right], \\
& S_{(n+1) h}=\sigma^{1-\vartheta}\left[S_{0 h}+\frac{r^{\vartheta}}{\Gamma(\vartheta+1)} \sum_{p=0}^{n} \omega_{n+1, p} y_{5}\left(t_{p}, \varphi_{p}\right)\right], \\
& I_{(n+1) h}=\sigma^{1-\vartheta}\left[I_{0 h}+\frac{r^{\vartheta}}{\Gamma(\vartheta+1)} \sum_{p=0}^{n} \omega_{n+1, p} y_{6}\left(t_{p}, \varphi_{p}\right)\right],
\end{aligned}
$$

so that $\omega_{n+1, p}=(n+1-p)^{9}-(n-p)^{9}$ and the functions $y_{j}$ for $j=0,1, \cdots, 6$ are expressed as:

$$
\begin{aligned}
y_{1}(t, \varphi(t))= & \Lambda_{k}-\beta_{1} S_{k}(t) I_{k}(t)-\beta_{2} S_{k}(t) I_{l}(t)-d_{k} S_{k}(t), \\
y_{2}(t, \varphi(t))= & \beta_{1} S_{k}(t) I_{k}(t)+\beta_{2} S_{k}(t) I_{l}(t)-d_{k} I_{k}(t), \\
y_{3}(t, \varphi(t))= & \Lambda_{l}-\beta_{3} S_{l}(t) I_{k}(t)-d_{l} S_{l}(t), \\
y_{4}(t, \varphi(t))= & \beta_{3} S_{l}(t) I_{k}(t)-d_{l} I_{l}(t), \\
y_{5}(t, \varphi(t))= & \Lambda_{h}-\beta_{4} S_{h}(t) I_{k}(t)-\beta_{5} S_{h}(t) I_{h}(t) \\
& -\beta_{6} S_{h}(t) I_{h}(t)-d_{h} S_{h}(t), \\
y_{6}(t, \varphi(t))= & \beta_{4} S_{h}(t) I_{k}(t)+\beta_{5} S_{h}(t) I_{l}(t) \\
& +\beta_{6} S_{h}(t) I_{h}(t)-d_{h} I_{h}(t) .
\end{aligned}
$$

5.2. Simulation. In the present subsection, we present a numerical simulation to investigate the transmission of 

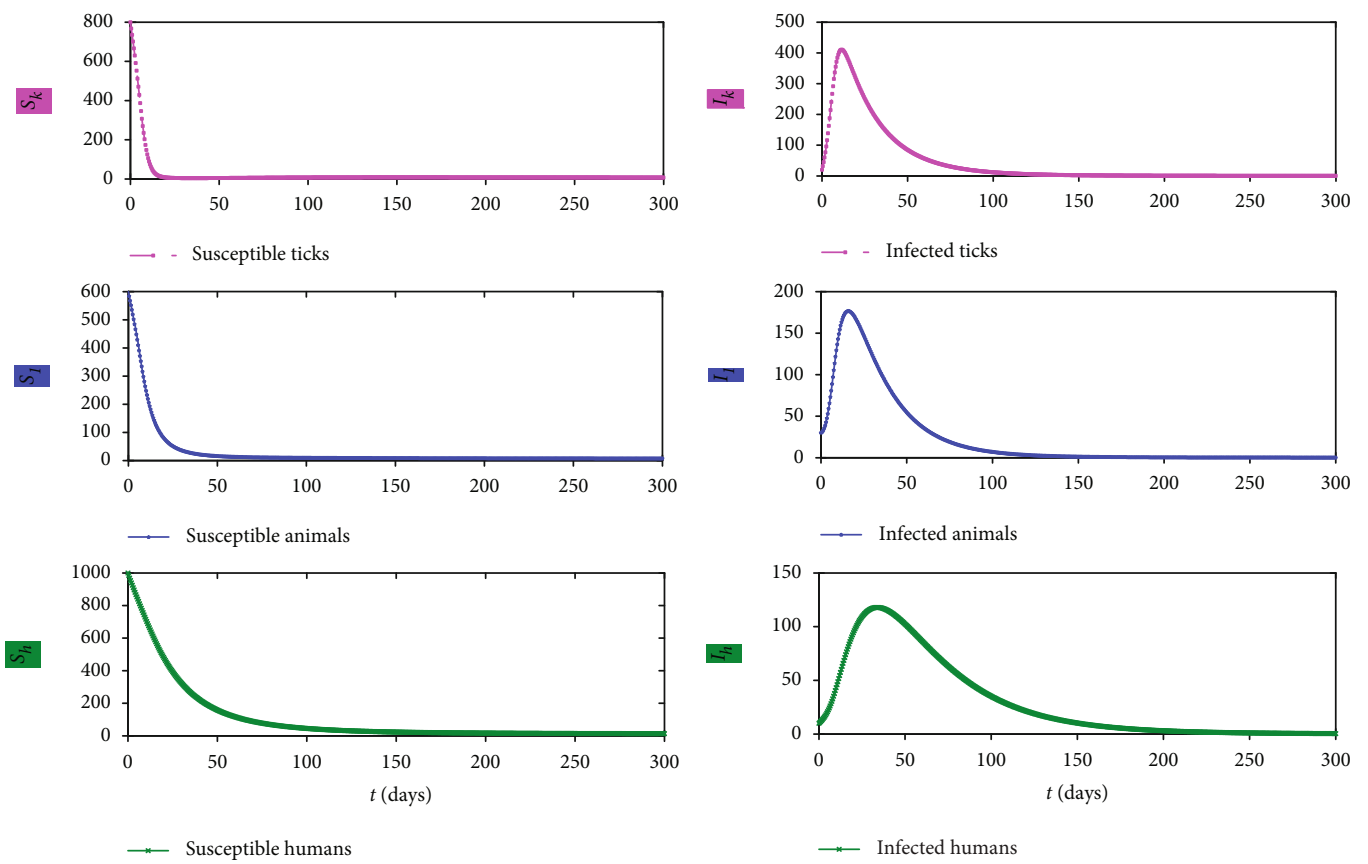

FIGURE 1: Plots of the results of model (10) with $R_{0}<1$ for $\vartheta=0.98$.

Crimean-Congo fever virus based on the amount of reproduction number. Also, we compare the results of the integer-order and fractional-order models.

To perform the desired simulation, in two cases, we consider different values for the parameters. In the first case, we assume: $\beta_{1}=0.5 \times 10^{-4}, \beta_{2}=0.3 \times 10^{-4}, \beta_{3}=0.1$ $\times 10^{-3}, \beta_{4}=0.03 \times 10^{-4}, \beta_{5}=0.4 \times 10^{-4}, \beta_{6}=0.7 \times 10^{-4}, \Lambda_{k}$ $=0.6, \Lambda_{l}=0.3, \Lambda_{h}=0.6, d_{k}=0.09, d_{l}=0.07, d_{h}=0.007, \sigma=$ 0.99 . We also consider the initial values as $S_{k}=800, I_{k}$ $=20, S_{l}=600, I_{l}=30, S_{h}=1000, I_{h}=10$.

Using the above parameters, we obtain $R_{h}=0.0233, R_{k l}$ $=0.00597$; thus, $R_{0}=0.0233<1$. Figure 1 shows the results of model (10) for all six groups for $\vartheta=0.98$. In this case, $R_{0}<1$, and Figure 1 shows that over time, the number of susceptible people is decreased, and the number of infected people is increased, but in less than 20 days, the number of infected people is decreased and eventually reaches zero, and the spread of the disease stops. In this case, $S(t)$ and $I(t)$ converge to the disease-free equilibrium point $E_{0}$.

In the second case, we assume that the disease transmission rate increases from the susceptible group to the infected group, and the transmission rates are equal to $\beta_{1}=0.5 \times 1$ $0^{-3}, \beta_{2}=0.3 \times 10^{-3}, \beta_{3}=0.1 \times 10^{-2}, \beta_{4}=0.7 \times 10^{-3}, \beta_{5}=0.4$ $\times 10^{-3}, \beta_{6}=0.7 \times 10^{-3}$. With these transfer rates, the value of the reproduction number is equal to $R_{0}=1.435>1$. Figure 2 shows the results of model (10) for the six groups studied in this case. Over time, the population of susceptible groups decreases and the population of infected groups increases, and finally, after 100 days, the population of infected groups decreases and converges to the endemic equilibrium point. As the rate of disease transmission increases, the value of $R_{0}$ increases, and we observe that the disease does not go away and its spread continues.
In this work, we have used the fractional-order derivative for modeling. In order to investigate the effect of derivation order, we have drawn the model results for infected groups with derivatives with integer-order $\vartheta=1$ and fractionalorder $9=0.98$ in Figure 3. Figure 3 shows that the results of model (10) are similar for the integer-order and the Caputo fractional order, and a small change in the order of derivation has no effect on the overall trend of the results in terms of ascending and descending, but the resulting numerical values are different.

\subsection{The Reproduction Number Sensitivity Analysis. We inves-} tigate the effect of parameters in Crimean-Congo hemorrhagic fever fractional model (10) on reproduction number using the method introduced by [40]. For this simulation, we use the parameters in the first case of the previous subsection. Since $R_{0}$ is defined as $R_{0}=\max \left(R_{h}, R_{k l}\right)$, therefore, we analyze the sensitivity of $R_{0}$ in two cases.

First, if $R_{0}=R_{h}$, by the mentioned method, we have $S_{\beta_{6}}$ $=\left(\partial R_{0} / \partial \beta_{6}\right)\left(\beta_{6} / R_{0}\right)=1>0, S_{\Lambda_{h}}=\left(\partial R_{0} / \partial \Lambda_{h}\right)\left(\Lambda_{h} / R_{0}\right)=1>$ $0, S_{d_{h}}=\left(\partial R_{0} / \partial d_{h}\right)\left(d_{h} / R_{0}\right)=-2 \beta_{6} \Lambda_{h} / d_{h}^{3}=-1.55<0$. Figure 4 shows the sensitivity of $R_{0}$ with respect to each of the parameters. As you can see, changing each of the parameters of model (10) that is involved in $R_{h}$ changes the reproduction number. The reproduction number is directly related to parameters $\beta_{6}, \Lambda_{h}$ and inversely related to parameter $d_{h}$. From an epidemiological point of view, whenever the reproduction number decreases, then the spread of the disease is controlled. Given that $\beta_{6}$ has the most positive effect on the $R_{0}$, so to control the spread of the disease, $\beta_{6}$ should be reduced through the reduced communication of infected and susceptible humans. 

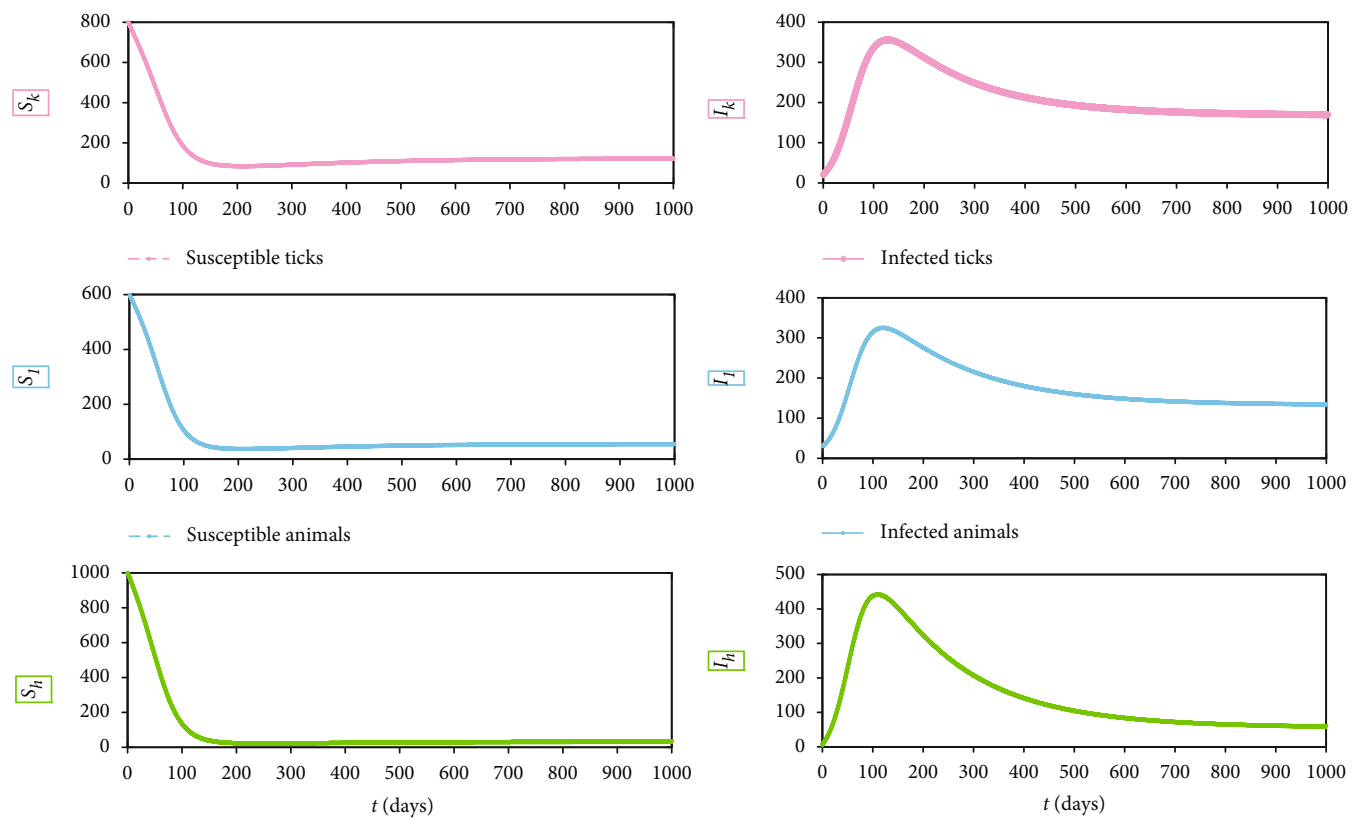

- Susceptible humans

- Infected humans

FIGURE 2: Plots of the results of model (10) with $R_{0}>1$ for $\vartheta=0.98$.
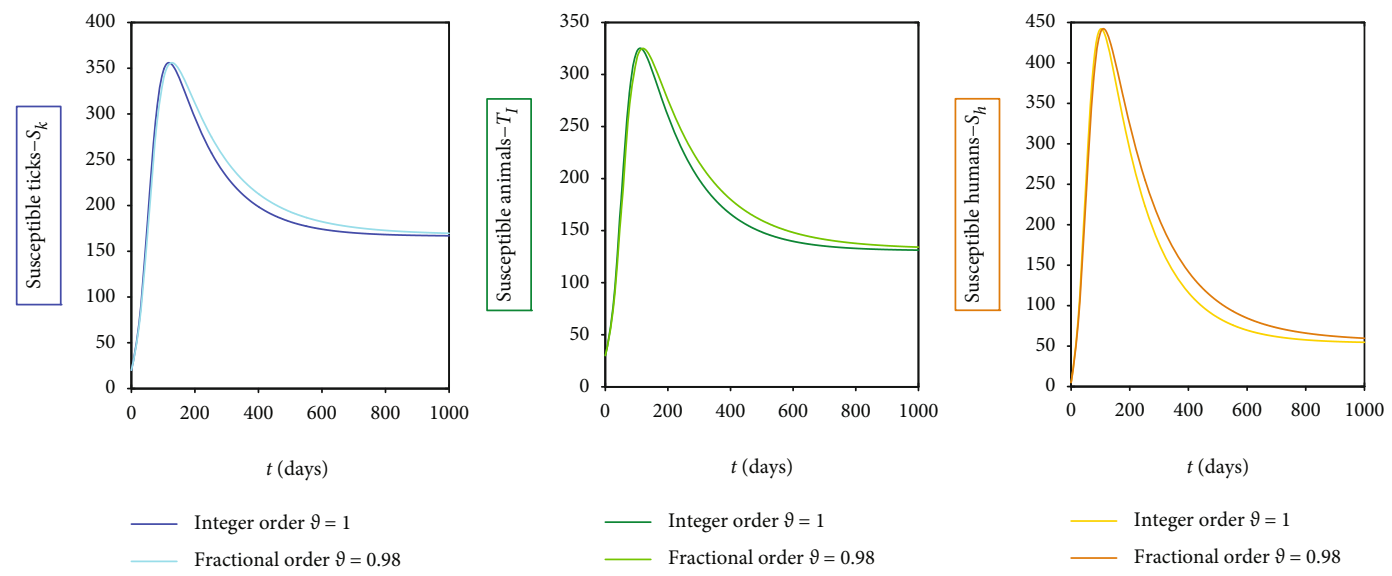

FIGURE 3: Plots of the results of model (10) for infected groups with integer-order $\vartheta=1$ and fractional-order $\vartheta=0.98$ in the case $R_{0}>1$.
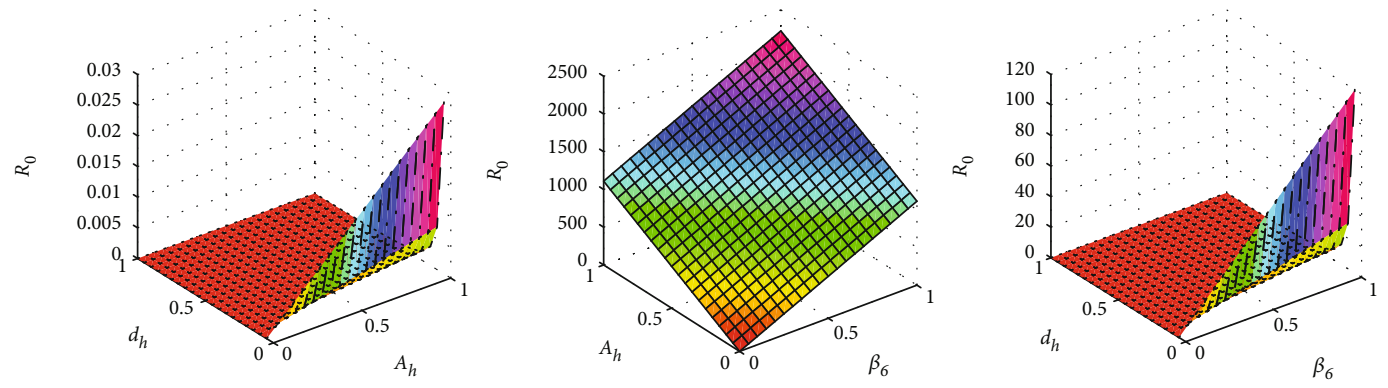

FIgURE 4: The graphs show the effect of model parameters on the reproduction number for the case $R_{0}=R_{h}$. 

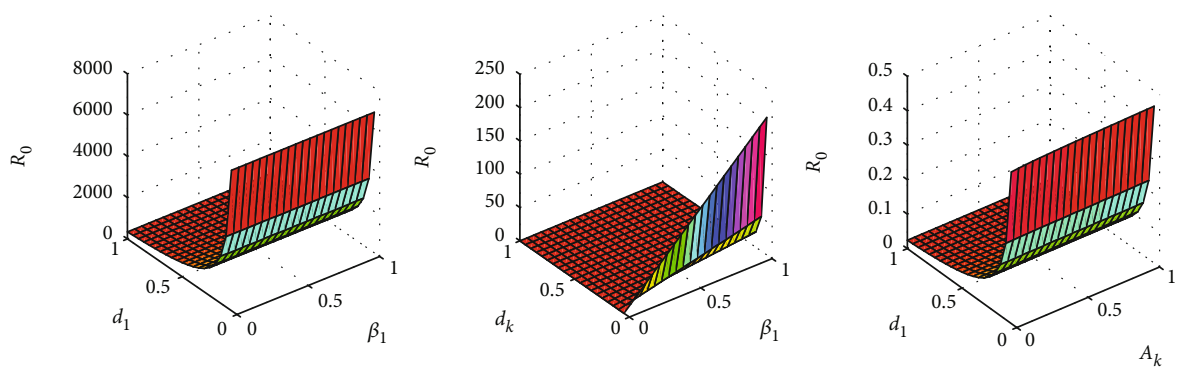

FIGURE 5: The graphs show the effect of model parameters on the reproduction number for the case $R_{0}=R_{k l}$.

In the latter case if $R_{0}=R_{k l}$, we obtain the same equations as above

$$
\begin{aligned}
S_{\Lambda_{k}} & =\frac{\partial R_{0}}{\partial \Lambda_{k}} \frac{\Lambda_{k}}{R_{0}}=0.724>0, S_{\beta_{1}}=\frac{\partial R_{0}}{\partial \beta_{1}} \frac{\beta_{1}}{R_{0}}=0.4486>0, \\
S_{d_{l}} & =\frac{\partial R_{0}}{\partial d_{l}} \frac{d_{l}}{R_{0}}=-0.5513<0, \\
S_{\beta_{2}} & =\frac{\partial R_{0}}{\partial \beta_{2}} \frac{\beta_{2}}{R_{0}}=0.275>0, S_{\beta_{3}}=\frac{\partial R_{0}}{\partial \beta_{3}} \frac{\beta_{3}}{R_{0}}=0.276>0, \\
S_{d_{k}} & =\frac{\partial R_{0}}{\partial d_{k}} \frac{d_{k}}{R_{0}}=-1.448<0 .
\end{aligned}
$$

Figure 5 shows the sensitivity of $R_{0}$ with respect to each of the parameters. The reproduction number is directly related to parameters $\beta_{1}, \beta_{2}, \beta_{3}$, and $\Lambda_{k}$ and inversely related to parameter $d_{l}, d_{k}$. Among the mentioned parameters, parameters $\beta_{1}, \beta_{2}$, and $\beta_{3}$ can be controlled, and all of which have a positive effect on causality, so to reduce the amount of reproduction number, it is enough to reduce the rate of disease transmission between ticks, animals, and humans.

\section{Conclusion}

In this work, we have presented a box model using the Caputo fractional-order derivative by taking into account the transmission of the Crimean-Congo hemorrhagic fever virus between ticks, animals (domestic and wild), and humans. We have calculated the feasible region and the equilibrium points of the system (10), and we have determined the necessary conditions for the stability of the equilibrium point. In the last section, using the Euler method for the Caputo fractional derivative, we have obtained the approximate solution of system (10), and then, we have provided a numerical simulation for the transmission of CrimeanCongo hemorrhagic fever virus. In two cases: $R_{0}<1$ and $R_{0}$ $>1$, the results of the model have been plotted for the six groups in the model, which clearly show that in the case $R_{0}$ $<1$, the transmission of the disease stops after a while, and the results of the system converge to the disease-free equilibrium point. We have increased the rate of disease transmission among the groups, and in this case, the results for $R_{0}>1$ show that the disease continues endemically, and also, the results converge to the endemic equilibrium point. The results of the model are compared with two types of deriva- tives of integer-order and fractional-order, and the result of comparison shows that changing the type of derivative with close order has no effect on the overall trend of the results but the obtained numerical values are different.

Later, we have investigated the effect of each of the model parameters on $R_{0}$, and the results show that the disease transmission rates among the groups have a positive effect on the value of $R_{0}$; therefore, to control the spread of CrimeanCongo hemorrhagic fever, the disease transmission rate should be reduced by reducing contact between different groups.

\section{Data Availability}

No data were used to support this study.

\section{Conflicts of Interest}

The authors declare that they have no competing interests.

\section{Authors' Contributions}

The authors declare that the study was realized in collaboration with equal responsibility. All authors read and approved the final manuscript.

\section{Acknowledgments}

The fifth author was supported by the Azarbaijan Shahid Madani University. The first author was supported by the Miandoab Branch of Islamic Azad University. Also, the third author was supported by the Prince Sultan University.

\section{References}

[1] O. Ergonul, "Crimean-Congo haemorrhagic fever," The Lancet Infectious Diseases, vol. 6, no. 4, pp. 203-214, 2006.

[2] J. C. Morrill, "Crimean-Congo hemorrhagic fever: a global perspective," Vector Borne and Zoonotic Diseases, vol. 9, 2008.

[3] M. Mardani and M. Keshtkar-Jahromi, "Crimean-Congo hemorrhagic fever," Archives of Iranian Medicine, vol. 10, no. 2, pp. 204-214, 2007.

[4] M. P. Chumakov, S. E. Smirnova, and E. A. Tkachenko, "Relationship between strains of Crimean haemorrhagic fever and Congo viruses," Acta Virologica, vol. 14, no. 1, pp. 82-85, 1970.

[5] N. Kuljic-Kapulica, "Emerging diseases. Crimean-Congo hemorrhagic fever," Medicinski Pregled, vol. 57, no. 9-10, pp. 453-456, 2004. 
[6] R. Swanepoel, D. E. Gill, A. J. Shepherd, P. A. Leman, J. H. Mynhardt, and S. Harvey, "The clinical pathology of Crimean-Congo hemorrhagic fever," Reviews of Infectious Diseases, vol. 11, Supplement_4, pp. S794-S800, 1989.

[7] T. Kurata, "Crimean-Congo hemorrhagic fever," Ryoikibetsu Shokogun Shirizu, vol. 23, Part 1, pp. 94-96, 1999.

[8] O. Ergonul, "Treatment of Crimean-Congo hemorrhagic fever," Antiviral Research, vol. 78, no. 1, pp. 125-131, 2008.

[9] P. Onguru, E. O. Akgul, E. Akınc1 et al., "High serum levels of neopterin in patients with Crimean-Congo hemorrhagic fever and its relation with mortality," The Journal of Infection, vol. 56, no. 5, pp. 366-370, 2008.

[10] A. Harxhi, A. Pilaca, Z. Delia, K. Pano, and G. Rezza, "Crimean-Congo hemorrhagic fever: a case of nosocomial transmission," Infection, vol. 33, no. 4, pp. 295-296, 2005.

[11] M. A. Çevik, A. Erbay, H. Bodur et al., "Clinical and laboratory features of Crimean-Congo hemorrhagic fever: predictors of fatality," International Journal of Infectious Diseases, vol. 12, no. 4, pp. 374-379, 2008.

[12] M. J. Erasmus, G. M. McGillivray, D. E. Gill et al., "Epidemiologic and clinical features of Crimean-Congo hemorrhagic fever in southern Africa," The American Journal of Tropical Medicine and Hygiene, vol. 36, no. 1, pp. 120-132, 1987.

[13] P. Nabeth, M. Thior, O. Faye, and F. Simon, "Human Crimean-Congo hemorrhagic fever, Sénégal," Emerging Infectious Diseases, vol. 10, no. 10, pp. 1881-1882, 2004.

[14] A. Kashkynbayev and D. Koptleuova, "Global dynamics of tickborne diseases," Mathematical Biosciences and Engineering, vol. 17, no. 4, pp. 4064-4079, 2019.

[15] K. Ergena, A. Cillib, and N. Yahniogluc, "Predicting epidemic diseases using mathematical modelling of SIR," Acta Physica Polonica A, vol. 128, no. 2-B, 2015.

[16] J. Switkes, B. Nannyonga, J. Y. T. Mugisha, and J. Nakakawa, "A mathematical model for Crimean-Congo haemorrhagic fever: tick-borne dynamics with conferred host immunity," Journal of Biological Dynamics, vol. 10, no. 1, pp. 59-70, 2016.

[17] M. M. Matar, M. I. Abbas, J. Alzabut, M. K. A. Kaabar, S. Etemad, and S. Rezapour, "Investigation of the p-Laplacian nonperiodic nonlinear boundary value problem via generalized Caputo fractional derivatives," Advances in Difference Equations, vol. 2021, no. 68, 2021.

[18] S. Etemad, M. S. Souid, B. Telli, M. K. A. Kaabar, and S. Rezapour, "Investigation of the neutral fractional differential inclusions of Katugampola-type involving both retarded and advanced arguments via Kuratowski MNC technique," Advances in Difference Equations, vol. 2021, no. 2014, 2021.

[19] J. Alzabut, A. Selvam, R. Dhineshbabu, and M. K. Kaabar, "The Existence, Uniqueness, and Stability Analysis of the Discrete Fractional Three-Point Boundary Value Problem for the Elastic Beam Equation,” Symmetry, vol. 13, no. 5, p. 789, 2021.

[20] T. Abdeljavad and D. Baleanu, "On fractional derivatives with exponential kernel and their discrete versions," Reports on Mathematical Physics, vol. 80, no. 1, pp. 11-27, 2017.

[21] H. M. Ahmed, R. A. Elbarkouky, O. A. M. Omar, and M. A. Ragusa, "Models for covid-19 daily confirmed cases in different countries," Mathematics, vol. 9, no. 6, p. 659, 2021.

[22] K. Muhammad Altaf and A. Atangana, "Dynamics of Ebola disease in the framework of different fractional derivatives," Entropy, vol. 21, no. 3, p. 303, 2019.

[23] M. Taghipoura and H. Aminikhah, "A new compact alternating direction implicit method for solving two dimensional time fractional diffusion equation with Caputo-Fabrizio derivative," Univerzitet u Nišu, vol. 34, no. 11, pp. 3609-3626, 2020.

[24] Z. Bouazza, S. Etemad, M. S. Souid, S. Rezapour, F. Martínez, and M. K. A. Kaabar, "A study on the solutions of a multiterm FBVP of variable order," Journal of Function Spaces, vol. 2021, Article ID 9939147, 9 pages, 2021.

[25] R. Almeida, A. M. C. Brito da Cruz, N. Martins, and M. T. T. Monteiro, "An epidemiological MSEIR model described by the Caputo fractional derivative," International Journal of Dynamics and Control, vol. 7, no. 2, pp. 776-784, 2019.

[26] D. Baleanu, H. Mohammadi, and S. Rezapour, "Analysis of the model of HIV-1 infection of CD4+ T-cell with a new approach of fractional derivative," Advances in Difference Equations, vol. 2020, no. 1, 2020.

[27] D. Baleanu, A. Jajarmi, H. Mohammadi, and S. Rezapour, "A new study on the mathematical modelling of human liver with Caputo-Fabrizio fractional derivative," Chaos, Solitons \& Fractals, vol. 134, article 109705, 2020.

[28] S. Rezapour and H. Mohammadi, "A study on the AH1N1/09 influenza transmission model with the fractional CaputoFabrizio derivative," Advances in difference equations, vol. 2020, no. $1,2020$.

[29] S. Rezapour, H. Mohammadi, and A. Jajarmi, "A new mathematical model for Zika virus transmission," Advances in difference equations, vol. 2020, no. 1, 2020.

[30] J. Singh, "Analysis of fractional blood alcohol model with composite fractional derivative," Solitons and Fractals, vol. 140, article 110127, 2020.

[31] J. Singh, D. Kumar, and D. Baleanu, "A new analysis of fractional fish farm model associated with Mittag-Leffler type kernel," International Journal of Biomathematics, vol. 13, no. 2, article 2050010, 2020.

[32] J. Singh, B. Ganbari, D. Kumar, and D. Baleanu, "Analysis of fractional model of guava for biological pest control with memory effect," Journal of Advanced Research, 2020.

[33] B. Ghanbari, D. Kumar, and J. Singh, "An efficient numerical method for fractional model of allelopathic stimulatory phytoplankton species with Mittag-Leffler law," Discrete and Continuous Dynamical Systems Series S, 2020.

[34] S. G. Samko, A. A. Kilbas, and O. I. Marichev, Fractional Integrals and Derivatives: Theory and Applications, CRC Press, 1993.

[35] M. Caputo and M. Fabrizio, "A new definition of fractional derivative without singular kernel," Progress in Fractional Differentiation and Applications, vol. 1, no. 2, pp. 73-85, 2015.

[36] J. Losada and J. J. Nieto, "Properties of the new fractional derivative without singular kernel," Progress in Fractional Differentiation and Applications, vol. 1, no. 2, pp. 87-92, 2015.

[37] M. Z. Ullah, A. K. Alzahrani, and D. Baleanu, “An efficient numerical technique for a new fractional tuberculosis model with nonsingular derivative operator," Journal of Taibah University for Science, vol. 13, no. 1, pp. 1147-1157, 2019.

[38] P. Van den Driessche and J. Watmough, "Reproduction numbers and sub-threshold endemic equilibria for compartmental models of disease transmission," Mathematical Biosciences, vol. 180, no. 1-2, pp. 29-48, 2002.

[39] C. Li and F. Zeng, "The finite difference methods for fractional ordinary differential equations," Numerical Functional Analysis and Optimization, vol. 34, no. 2, pp. 149-179, 2013.

[40] T. Khan, Z. Ullah, N. Ali, and G. Zaman, "Modeling and control of the hepatitis B virus spreading using an epidemic model," Chaos, Solitons \& Fractals, vol. 124, pp. 1-9, 2019. 